\title{
A participatory design research method using Chinese character experiments: using a range of participants to test the potential readability of new characters
}

\author{
Wenbo Ai, Royal College of Art \\ wenbo.ai@network.rca.ac.uk
}

\begin{abstract}
The author of this paper is an Mphil student studying at the Royal College of Art, School of Communication. His research topic concerns low literacy and literacy design and has two aims: firstly, to determine whether design language as a cognitively functional system can cross cultural and linguistic barriers to facilitate illiterate cognition. Secondly, to ask whether data achieved and any co-designed research findings can usefully reflect on and contribute to design literacy? The paper describes three experiments conducted at the School of Communication Research Work in Process Show, in December 2017. These experiments are intended as catalysts: to see what the challenges of the researcher's research project on levels of literacy and design might be, to explore participant strategies across barriers of culture and literacy, to format appropriate materials and methods which could contribute to his research journey.
\end{abstract}

Keywords: Participatory methodology, Chinese Typeface design 


\section{Research Question and Background}

The first aim was to design and test visual material tools that could help speakers from any cultural background understand - at least in part - the ways in which Chinese characters have been derived. Although some participants were Chinese speakers their responses were often deeply programmed by their linguistic cultures. The reasoning behind the three experiments was to explore a participant design research method using Chinese characters which hybridises other visual languages, images, pictographs and icons. One of the hypotheses here lays the theoretical groundwork for a new character design, which integrates other visual language, image, pictogram and icon, could facilitate literate cognition. Hypothetically, the strategies by which people see, recognise and then use pictographs are culturally malleable. Are there lessons to be learnt for the design of user-centred information more broadly, in China and elsewhere?

The Chinese writing system is far from static: in fact, the script has continued to evolve from prehistoric times until the standardisations of the twentieth century. The earliest known form of Chinese writing, oracle bone script or 甲骨文, is more like an image rather than a character (Bo (2003)) and dates back to the Shang dynasty (more than $1000 \mathrm{BCE})^{1}$. Metal script or 金文 found on bronzes made in the Zhou dynasty (approximately $700 \mathrm{BCE}$ ) show further developments, while three hundred years later the Script of the Six States 六國文字/六国文字 became the precursor to Large Seal Script and Small Seal Script (Park (2016)). These appear more iconic in the way they represent objects - pictographs - or ideas - ideographs - (Han (2012)) and became standardised in the late Qin (approximately $200 \mathrm{BCE}$ ). It is from these efforts at standardisation that elements of modern Chinese characters appear (Norman (1988)). At the early stages, the strokes of a character may resemble the shape of an objects being represented in a pictograph. For ideas, greater abstraction was necessary because there was no clear association with a shape. When it comes to recognisably modern Chinese characters, however, the intimate pictographic connections between character and object symbolised become much weaker. Chinese writing has become - since the further standardisations of the late Ming and early Qing (between the fifteenth and seventeenth

\footnotetext{
${ }^{1}$ Essentially turtle shells or Ox bones had characters carved on them before being fired. The characters might be questions or postulates. The firing caused the bone to crack and these lattices then indicated answers (Norman (1988)). There has recently been debate about much earlier finds dating back to 5000 $\mathrm{BCE}$ and whether these indicate an even earlier origin of Chinese script.
} 
centuries) - more linear and stylised, with those characters that had been historically pictographic resembling the objects they identify more distantly or not at all. ${ }^{2}$

There has been discussion about the ways in which Chinese pictographs lost their direct symbolic associations at the end of an evolutionary process, especially from traditional to simplified Chinese (Li, (2003); Wang, (2010); Qian, (2013)). What has not been examined is the interference between pictographs and ideographs: it is possible that the abstractions of the latter weakened the clear associations of the former. It is also possible that aggregates and phonetic elements worked to dilute the pictographic. The relevance here to contemporary design is significant, and as yet there seems to be little if any analysis in this area: what are the systemic interference patterns between different elements of written Chinese? Could a better understanding allow design practitioners to generate more effective and more usable visual language?

In terms of the legibility of visual language, semiology and image have an advantage over characters. Chinese characters combine meaning and shape, however, the evolution - and the interferences between different functionalities - means the priority of pictogram has been lost. What will be the next evolution of the Chinese character and can a better modelling of previous changes help not only understanding but also parallel design developments? Can pictographic factors be re-absorbed into this process? Can a new and possibly more user-friendly Chinese type - one that integrates image, symbol/icon and pictograph - be designed; one that is more legible and even may facilitate cognition and learning for non-Chinese speakers who may be unaware of Chinese culture? Indeed, can such a system be a commutative tool for a universal language?

Since most participants in this study are non-Chinese and unfamiliar with Chinese characters, this process of modelling could contribute to better learning and greater communication. Designing a more recognisable Chinese character - combining pictographic, logical aggregate and phonetic elements - might contribute to better, more effective, user-oriented systems. These would occupy those places left vacant by designers and communicators, places occupied by those with low literacy (or none), or those who want to start to learn Chinese as an auxiliary language.

\footnotetext{
2 It should be noted that around $95 \%$ of Chinese characters are "logical aggregates" - where a single meaning is derived from two or more characters - or "phonetic complexes" where one element is used for its sound. The idea of image and the complex ways it is embodied in Chinese calligraphy, suggest dynamic semantic/calligraphic/phonetic relationships ( $\operatorname{Li}(2010))$.
} 
These elements - combining design, linguistic/calligraphic evolution and human needs - are what the researcher wants to explore through this typographic experiment. Meanwhile, exploring the participatory research method in typographic design - which could generalize more user-centred design work, in this case - the result of any design could be more intimate to audiences and their worlds. It could, to rephrase one description, get right into people's minds (Zender, (2017)) in the most generative way.

\section{Comparative studies}

In the 1930s, Neurath and Arntz designed an International System of Typographic Picture Education (ISOTYPE), their intention was transferring complex social facts pictorially to a legible and memorable visual language (Neurath (2010)). Later, they attempted to develop a universal visual system which would reach beyond differences in language and culture. ISOTYPE turned out, however, to be an assistant language not a verbal language. It became a visual education to help citizens understand information more attractively and accurately - with a greater degree of validity. Most importantly, ISOTYPE is a pioneer in the exploration of universal language in the visual communication design field.

Following the ISOTYPE paradigm, a number of design research studies have focused on cross-cultural communication, pictographics, icons and visual images: this approach has dramatically spread through multiple and not always obviously associated disciplines. Originally, the designers received the requirements and a commitment from clients and then designed outcomes based on their understanding and experiences. After the work was agreed, the design work would be published, and users became automatically new learners, adopting the new designs. To improve the comprehensibility and practicability of design, the roles of designers and the design process itself have been changed dramatically. Design principles have driven outcomes to be more user-centered in most design fields. In the visual communication design area, more and more pictographically-based design studies turned to user drawing and other participatory design methods (Strauss and Zender, (2017); Paulovich, (2015)) and to derived participatory design (Bennett, (2015)). These study cases have proved that the role of the visual communication designer has changed, and participation with users can be a starting point for design projects to gain better and wider understanding (Barnes, 
Taffe and Miceli, (2009)). After all, a designer needs to be a participator with the user, communicator to and from the user, an observer of people before they can embark on any project.

Key study cases have particularly demonstrated this need. Strauss and Zender (2017) highlight the importance of "consensus analysis" as a new method for designing effective pictograms. Their method involves a thorough data collection from participants focused on three hospital pictograms, exploring what elements combined could represent "oncology", "outpatients" and "psychiatry." They aim for consensus before starting to design any pictograms. Consensus analysis "specifies the conditions under which agreement between people can be seen as a sign of knowledge or getting it right" (Borgatti and Halgin, (2011)) and, even more importantly, such analysis "provides an empirical means by which a researcher can measure and describe the cultural knowledge of participants in a study" (Romney, Weller and Batchelder, (1986)). In order to maximize the users' comprehension and therefore design more effective pictograms, collecting data from users and understanding what they expect to see in a pictogram is essential before even a rough draft of the pictogram is created (Strauss and Zender, (2017)). Zender also points out that a person's visual cognition depends on memory, education and cultural background: these factors may be more or less fixed and reflect on how a pictogram is decoded or how people might express or draw a pictogram if asked to participate (Zender, (2017)).

Wang (2010) experiments using two factors - alphabetic or non-alphabetic representations and cultural or standard imagery - which relate to icon representations that affect Taiwanese computer users. Results indicate that icons with alphabeticcultural elements are perceived better than western standard icons by participants not familiar with computers. Another project examining the delivery of safer sex practices in Ghana uses a participatory method to localize the denotative elements of an image to improve user experience and cross-cultural usability (Bennett, (2015)).

These studies show that knowledge and cultural understanding may determine perspective and cognition, though research so far does not make the overall cognitive mechanisms clear. Consensus analysis provides an opportunity to go further and decode cognitive activity before designers embark on design processes. Designers may then use decoded results to formalize a more used-centered outcome. How to decode cognitive processes successfully in an unbiased manner is, however, another issue which needs to 
be carefully considered. Specific and appropriate design material could channel consensus analysis, but this also needs to be thoroughly prepared. All these previous studies provide a generative theoretical and practical base and contribute to the experimental methodology of the next stage.

\section{Experimental methodology}

The experiment had three parts: the first was made up of several small tests to assess the appropriate design method and materials to use for the show; the second was a workshop, presented at the RCA School of Communication Research Work in Process Show, and collected the data for the final design; the ultimate part was a design outcome based on the observational studies, data collection and experiments.

Designing an appropriate visual material tool - one that can be used to communicate using a card carrying a design - to facilitate and stimulate the process of comprehension is always essential to a project such as this. Material tools need to be carefully designed to facilitate audiences' (or potential users') engagement during their visit. Before the exhibition started, in order to understand non-Chinese cognition of Chinese characters and achieve the best approach for the exhibition.

Several methods were tested:

- The first method was to ask non-Chinese simply to match Chinese characters with pictures, creating word-picture matching material to facilitate informational and cognitive processing (Noël, (2015)). It used in total 11 character cards, potentially corresponding to 11 pictures. During this playcentred game, participants had to guess and match character card to picture. This process unconsciously influenced their cognition of Chinese characters, especially their understanding of the attributes of pictographic characters. While it was fun it did not make any contribution to the next design stage.

Understanding pictographic attributes was not enough. What was needed was a new element to facilitate the understanding of further details of each character and stimulate their motivation into the next design stage. 


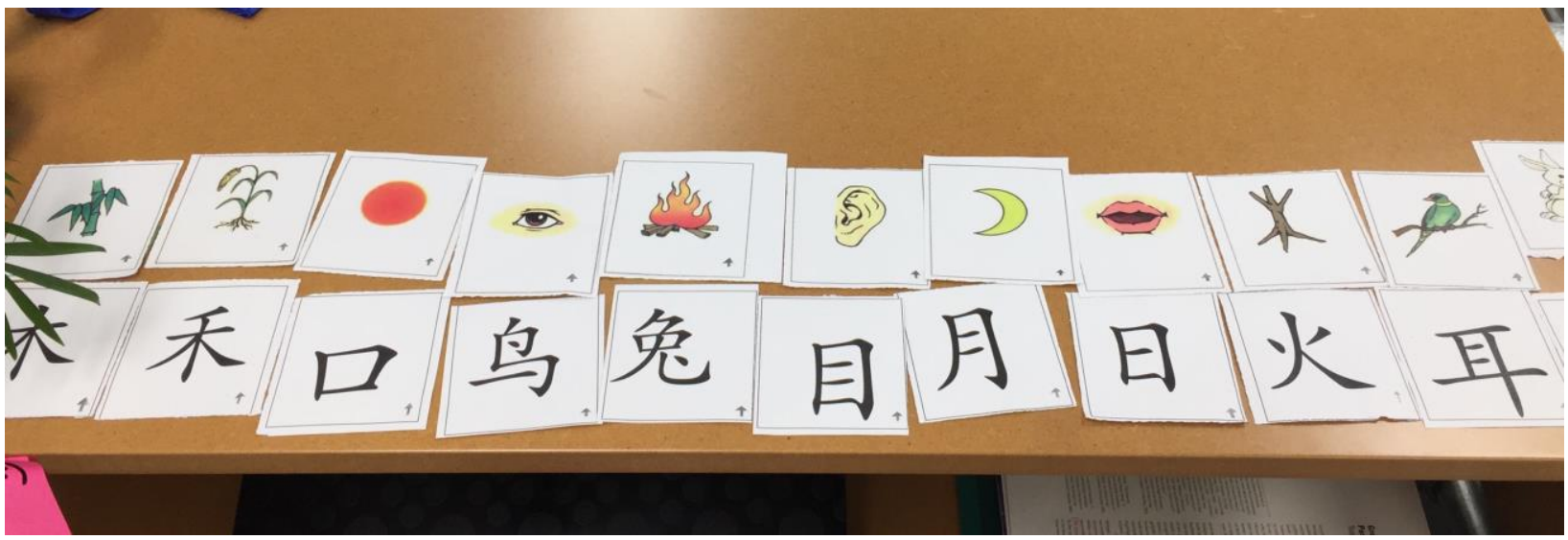

Figure 1: First design toolkit

- The second method was slightly less straightforward. The researcher added knowledge of the evolution of Chinese characters into this game. Six different types of Chinese Characters - Oracle Bone Script, Large Seal Script, Small Seal Script, Clerical Script, Semi-cursive Script, Cursive Script - are the most important historically. Using this evolutionary paradigm, it is clear how Chinese characters have changed from merely drawing a picture to writing an abstract character; how Chinese characters evolved from images into the complexity of modern Chinese (Li, 2014). This design therefore had five images: a person, rain, a fish, a mountain, a horse. Each image was represented by seven Chinese characters which included Oracle Bone Script, Large Seal Script, Small Seal Script, Clerical Script, Semi-cursive Script, Cursive Script and modem ChineseKai. Then cards were disrupted and participants were asked to try to match each image to its seven characters.

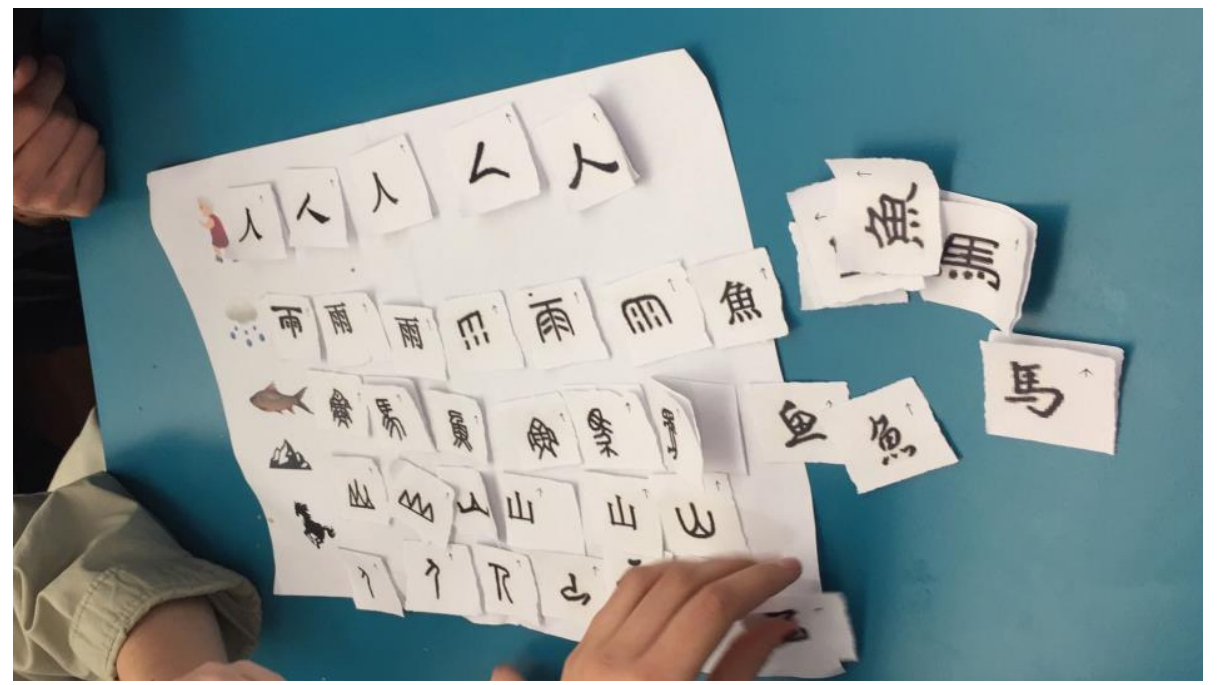

Figure 2: Matching six types of character to five images 
Feedback from the participants who had used both methods was the second design toolkit was more useful to understand the pictographic features of Chinese characters. Reducing the number of the objects - the character cards - and focusing on one, giving more time to allow a search through the different scripts, helped participants better understand the evolutionary process from drawing to writing. They admitted that the process of matching different scripts to images encouraged their connection between the strokes constituting each character and the shape of each object. They had continuously to examine both image and character, their eyes had to move between the two categories and this uninterrupted comparison, movement, thought and reflection may have improved cognition of Chinese characters in a shorter time period.

- These two tests and associated feedback allowed the formatting of the final design toolkit for the exhibition. To keep the task simpler and make it more appealing, only four characters were selected to be designed: a fish, a bird, a horse, and a goat. From the previous tests, animal-based characters were more popular and achieved more accurate results. Reducing the number of characters and focusing on animal-based characters made this task more attractive than the previous methods.

After this design toolkit was complete, the next stage was to determine how to represent these design materials and how to design an effective method, one that would encourage participation. Considering the previous audience experience in the exhibition, participants did not take long to figure out a complex intention from one task and move elsewhere. Therefore, the problem was how to make this appealing, encouraging, attractive and motivating, and to make assessment less tiring and more interesting and pleasant. For an exhibition, people generally do not have patience nor time enough to play the matching card games before they were finally invited to design a character. So, this assistant toolkit needs to be changed and improved.

Based on this research study and feedback from participants, the final co-design materials were four posters shown on the exhibition stand. Each poster contained an image of an animal and the evolution of the associated Chinese character. Posters were the co-design space, displayed publically and encouraging people to participant spontaneously and share their experience with others. The timeline - clearly showing the evolution process on the bottom of the posters - reminds readers how each character 
evolved from beginning to end, from pictograph to modern character. Before a participant started to take part in this design process, observing this evolutionary process could assist their visual cognition in terms of each character, and formulate the thinking process for the next creative stage. For the exhibition, the cards designed for using and reflecting were not first-hand material, due to the short times of individual visits. These became back-up materiel for those who showed deeper interest in this project and in the nature and history of Chinese characters.

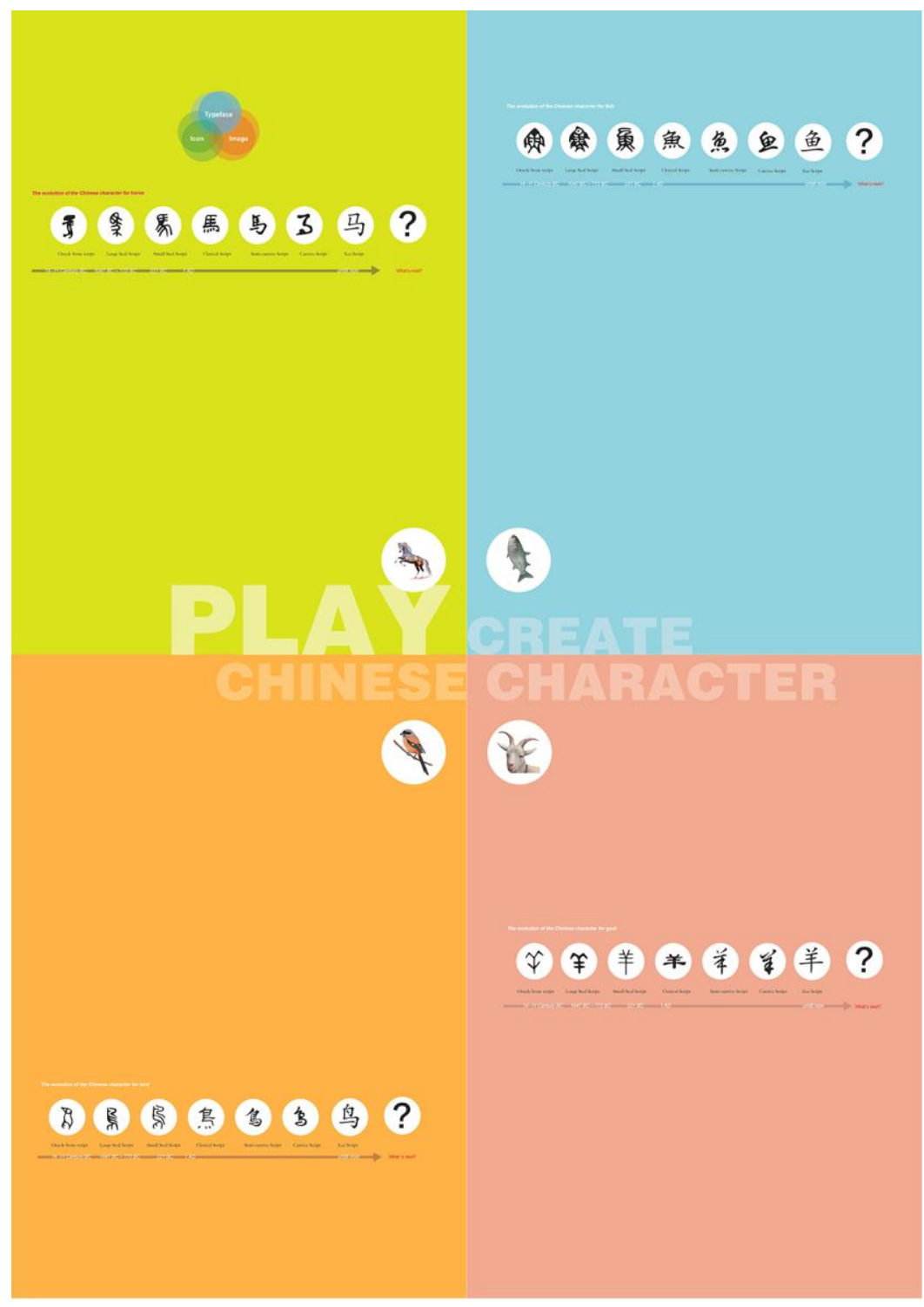

Figure 3: Posters designed to encourage people to participate 


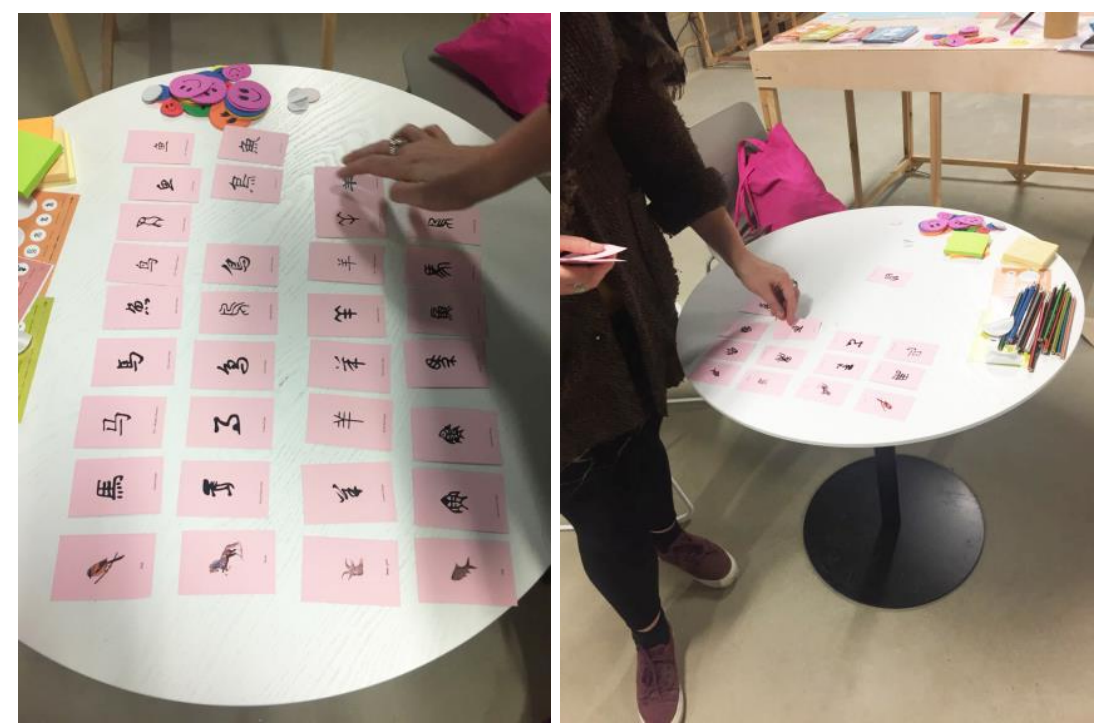

Figure 4: The matching game

It is now recognised that participants require a simple instruction for any task and that it be easy to follow. If these requirements are not met, participants disengage and the experiment fails (Song and Schwarz, (2009)). How to impart a basic understanding of Chinese characters so that a non-Chinese reader can gain familiarity with the characters, further motivating them to create something new in a short time was the first and biggest challenge. The solution was a label with a simple instruction:

Draw \& stick: please draw your Chinese characters based on the images (thinking about the process of their evolution) and stick the result on the posters. A free postcard for you to take, draw and share with friends.

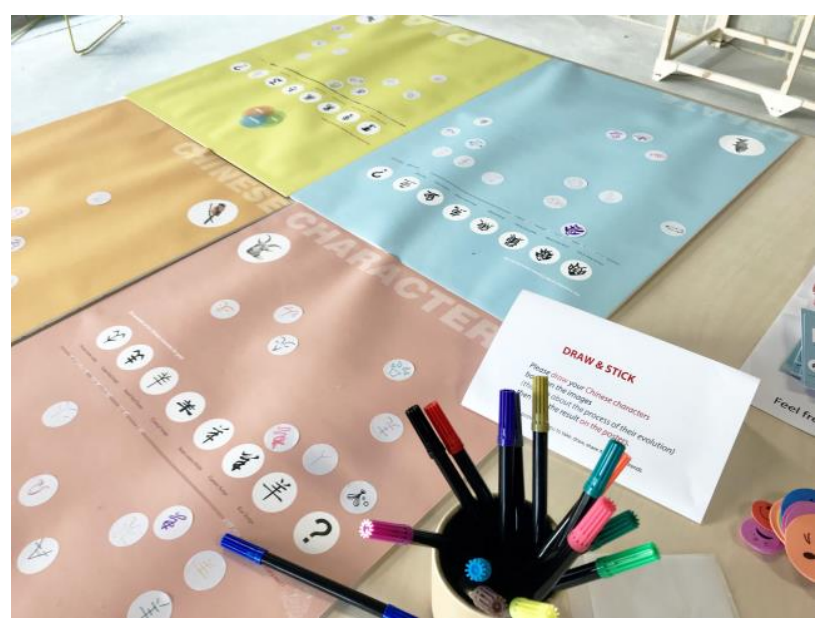

Figure 5: Instruction and presentation

\section{Ethical parameters}


One of the ethical issues involved in this project was how to avoid persuading people to participate in this co-design process, how to initiate a spontaneous behavioural response that was a key target for this workshop. Choosing to do this workshop during the RCA Research Work in Progress Show is a unique opportunity: admitting to attendant ethical issues was important. Visitors were aware that the workshop was part of each researcher's exploratory journey. They were aware that knowledge exchange and value sharing was the core of this activity.

To facilitate interaction - and perhaps minimise interference - all materials were designed to be as clear, simple and appealing as possible. With these parameters in place, the researcher could stand back and observe rather than step forward and persuade someone to participate and draw a character. When people passed by this exhibition stand, some were attracted by the colourful presentation and, after they had quickly worked out the target, whoever were interested in getting involved did so voluntarily and without prompting. They then were willing to move to the next stage. Most reactions after this point were similar: a participant would pick up a pen and a sticker - perhaps while others stood behind to observe the character evolution process, or compared stickers and discussed among themselves. Again, there was no need to prompt as these people then came forward and, after a few moments of observation and thought, started to draw a new character. The researcher did not at any time make his way into the participant or semi-participant/observant groups and explain anything: he remained an observer, standing at a distance from the display table (see figures 1, 2 and 3). There was only a level of (muted) involvement if a participant asked a question first and opened the conversation, perhaps in terms of the nature of Chinese characters. If a participant made it clear they were really interested in engaging with the Chinese characters then the researcher invited them to play "matching card" games. After these, some were willing to draw a new character as they felt they had understood sufficiently about character structure and pictographic attributes. Sometimes, the researcher gently reminded participants who were leaving to take postcards if they wanted (see figure 4). These interactive postcards were gifts to thank participants and contained shared values and knowledge to take away and share with friends. These postcards therefore became another co-design place, containing elements of the character evolution process. Users could draw a new character and send it to friends who, in turn, could draw another. The postcards can be seen as another link in the Chinese character knowledge exchange, 
expressing the insights participates took away from the exhibition, one that acknowledged engagement and curiosity on participants' terms.
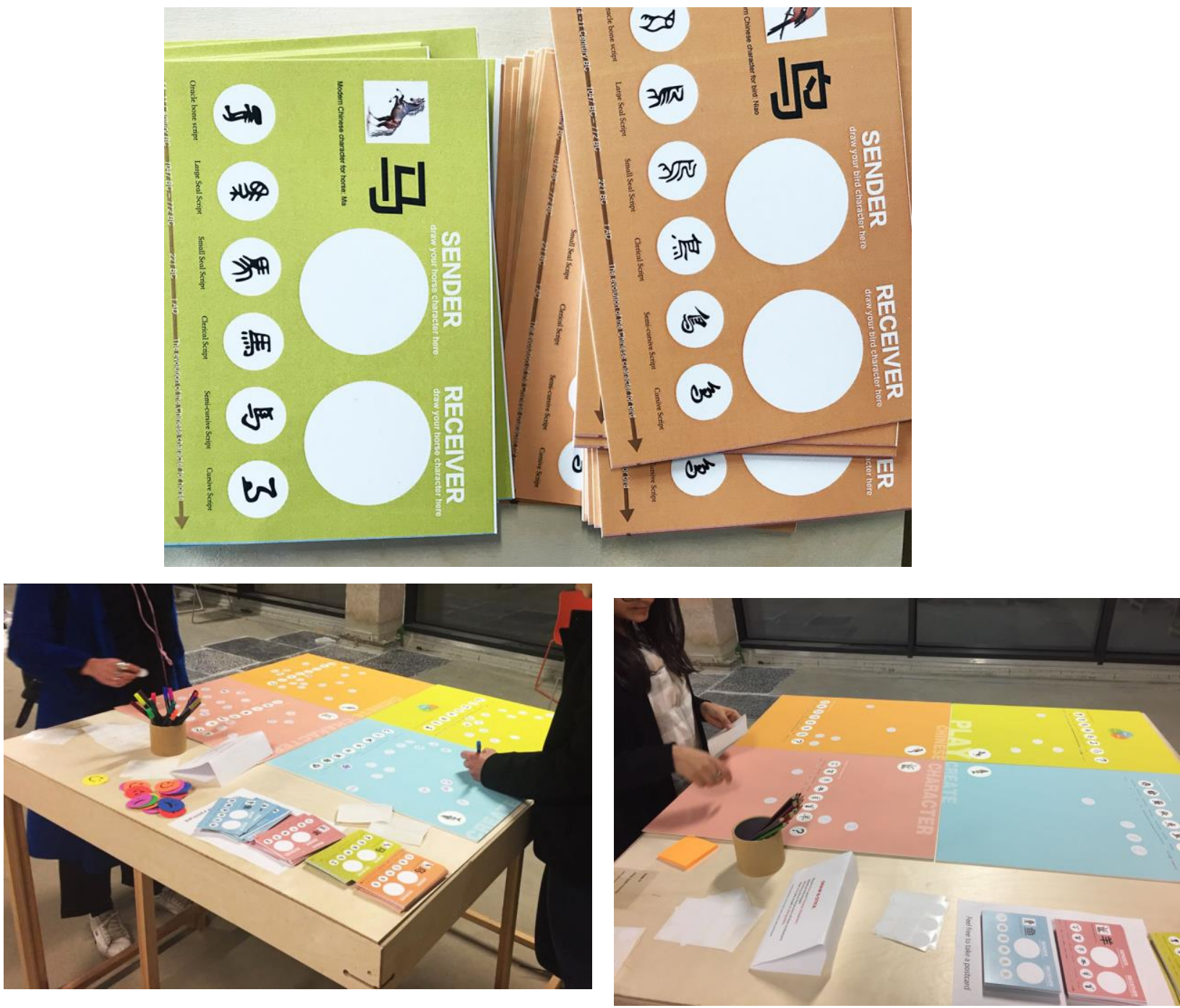

Figure 6: Participation and the postcards

None of the participants is readily identifiable, allowing participation to take place within as neutral a personal space as possible. Other ethical questions regard the parameters of cultural representation. For example, what are the ethics of redesigning a typeface? Regarding culturally-specific modes of representation, what are the ethics of getting people to look at different modes of representation typographically? Is this just part of the evolutionary process or might there be worries about putting identities under stress? In the case of this brief experiment what was being implied about respect - or otherwise - for the traditions represented in language was minimal. Had the experiment been more intense and used more Chinese indigenous speakers, then there might have 
been ethical issues derived from their perception that their culture was somehow being questioned.

\section{Results}

\section{Goat:}

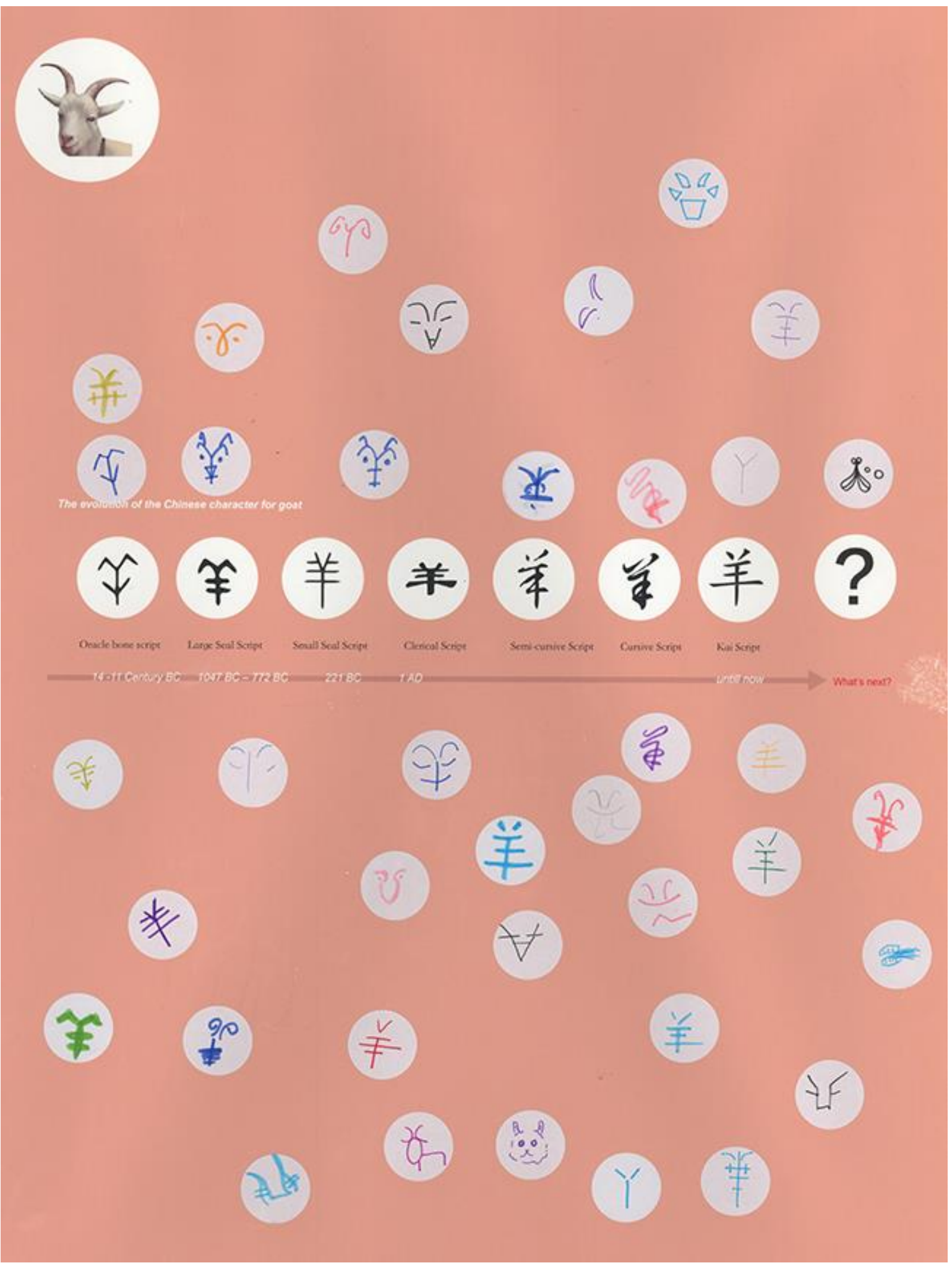




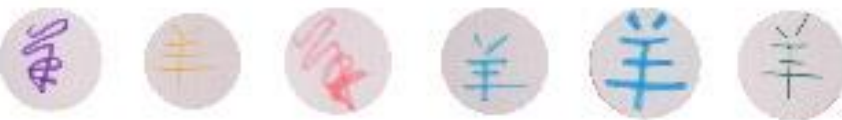

failed thetask, copied the words (6)

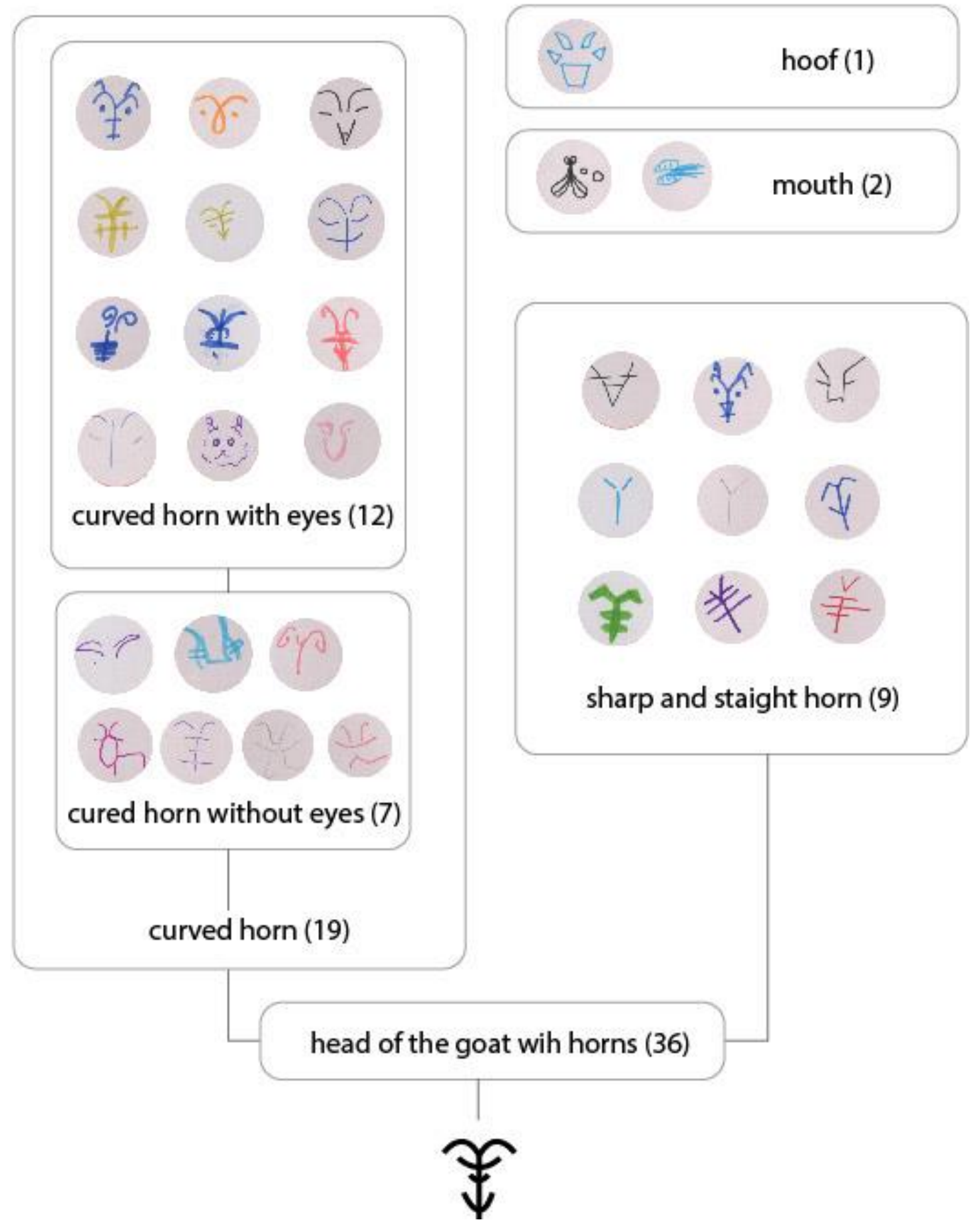

Figure 7: The goat poster with responses 
There were a total of 38 drawings stuck to the poster of goat. 36 were the head of the goat with horns; one showed a hoof, and two a mouth. Clearly, the horns were considered the main features, combining image with character. Within these 36 drawings showing horns, 7 had a V-shape, sharp and straight up; 12 were curved. Also, 15 participants drew the eyes of the goat. From the drawings, two salient features therefore emerged: the horns and the eyes. These participants captured from both images and characters and the two features were added to their final design concepts.

\section{Bird:}

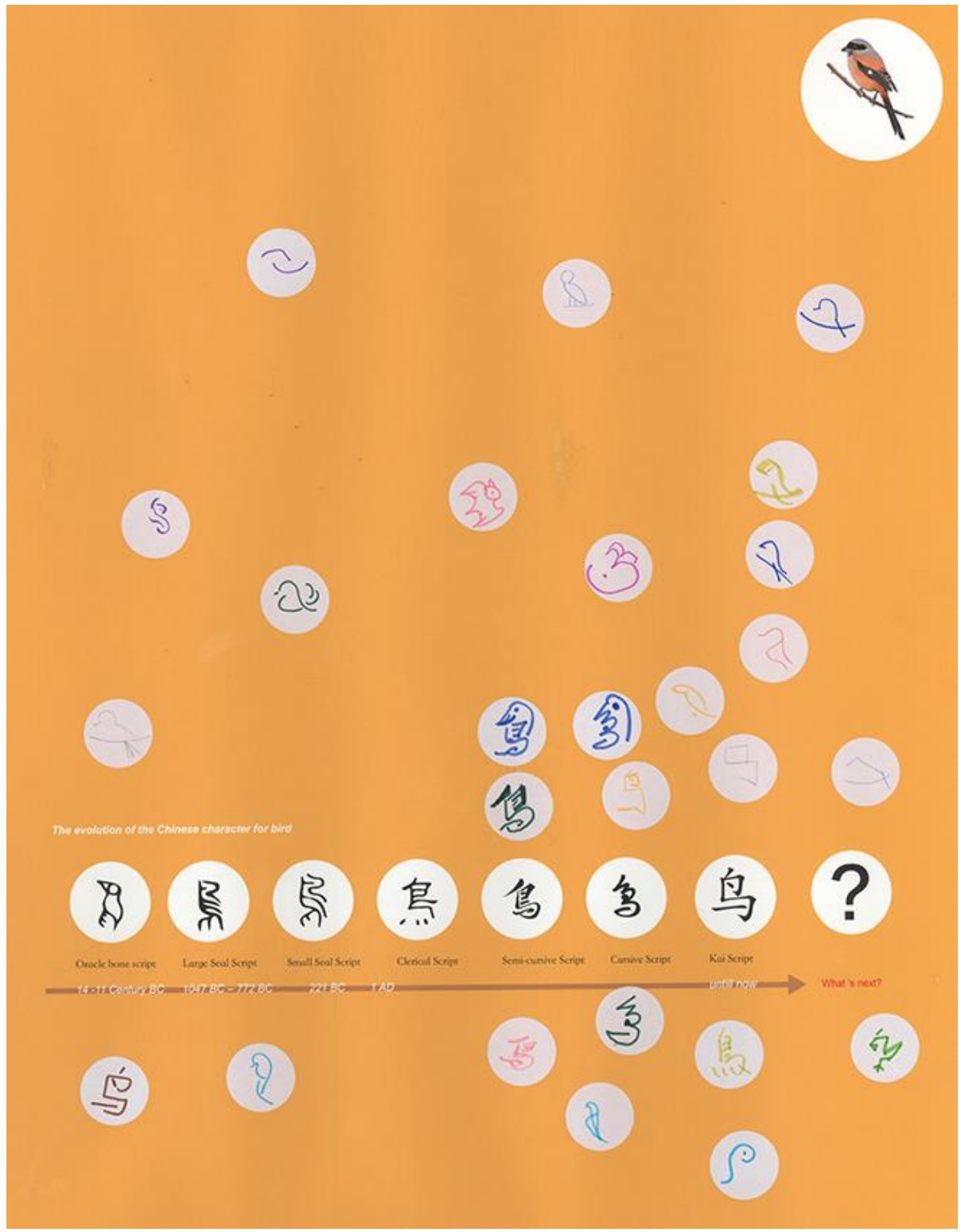




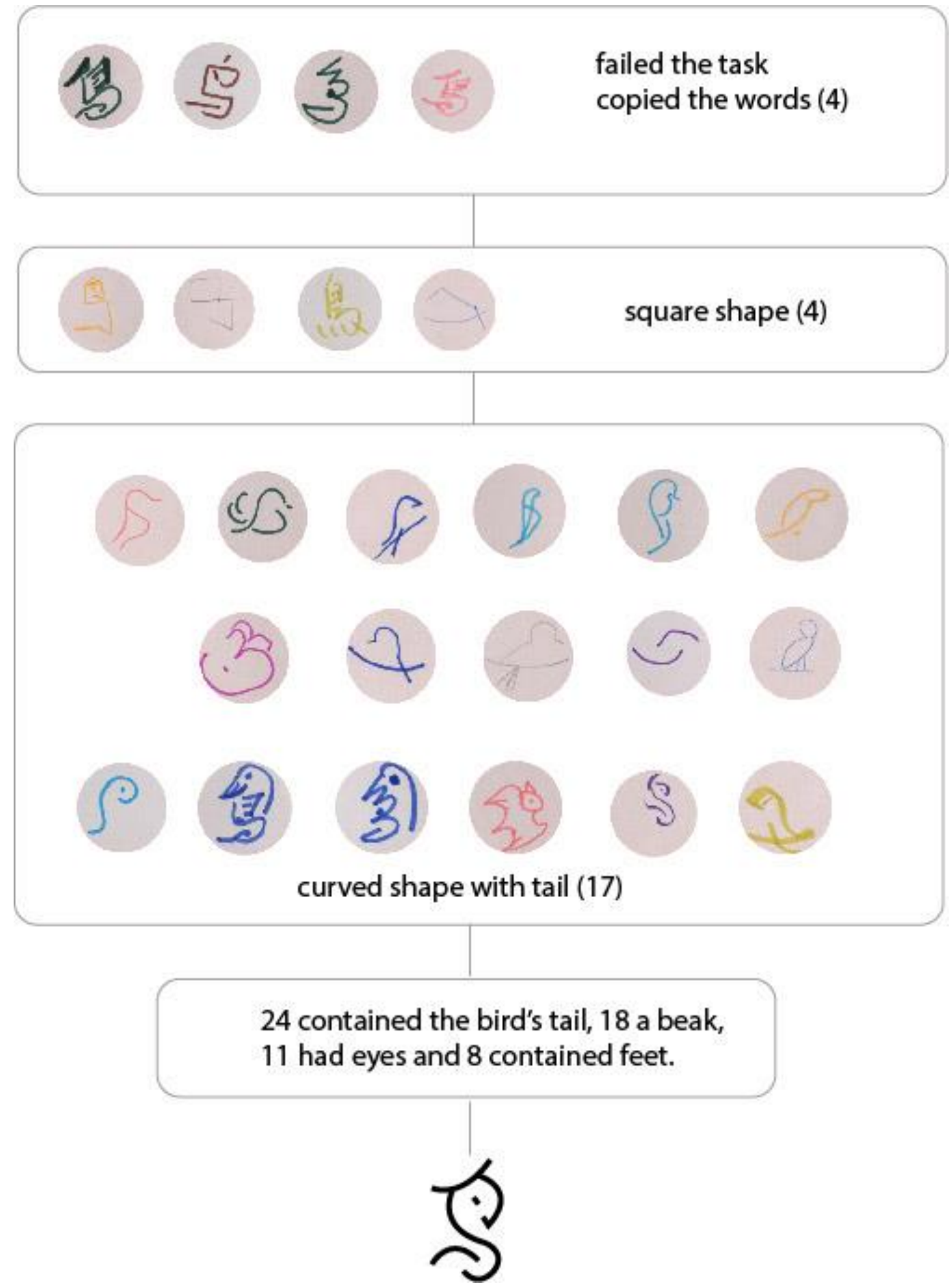

Figure 8: The bird poster with responses

26 birds are shown on this poster. Though the shape of the modern bird character looks more square than curved, 22 of the shapes were drawn curved, only 4 were square. This may be due to the common image of a bird as sinuous and moving not stiff and lifeless. Flight is, after all, the acme of gracefulness. 24 contained the bird's tail, 18 
a beak, 11 had eyes and 8 contained feet. Each of these elements can be found somewhere in the evolution of the Chinese characters for bird, up until the present. From the participants' results, curved lines plus tail, beak and eyes make up a bird. These are the common features which participants discovered from both images and characters and are considered in the final design.

\section{Fish:}

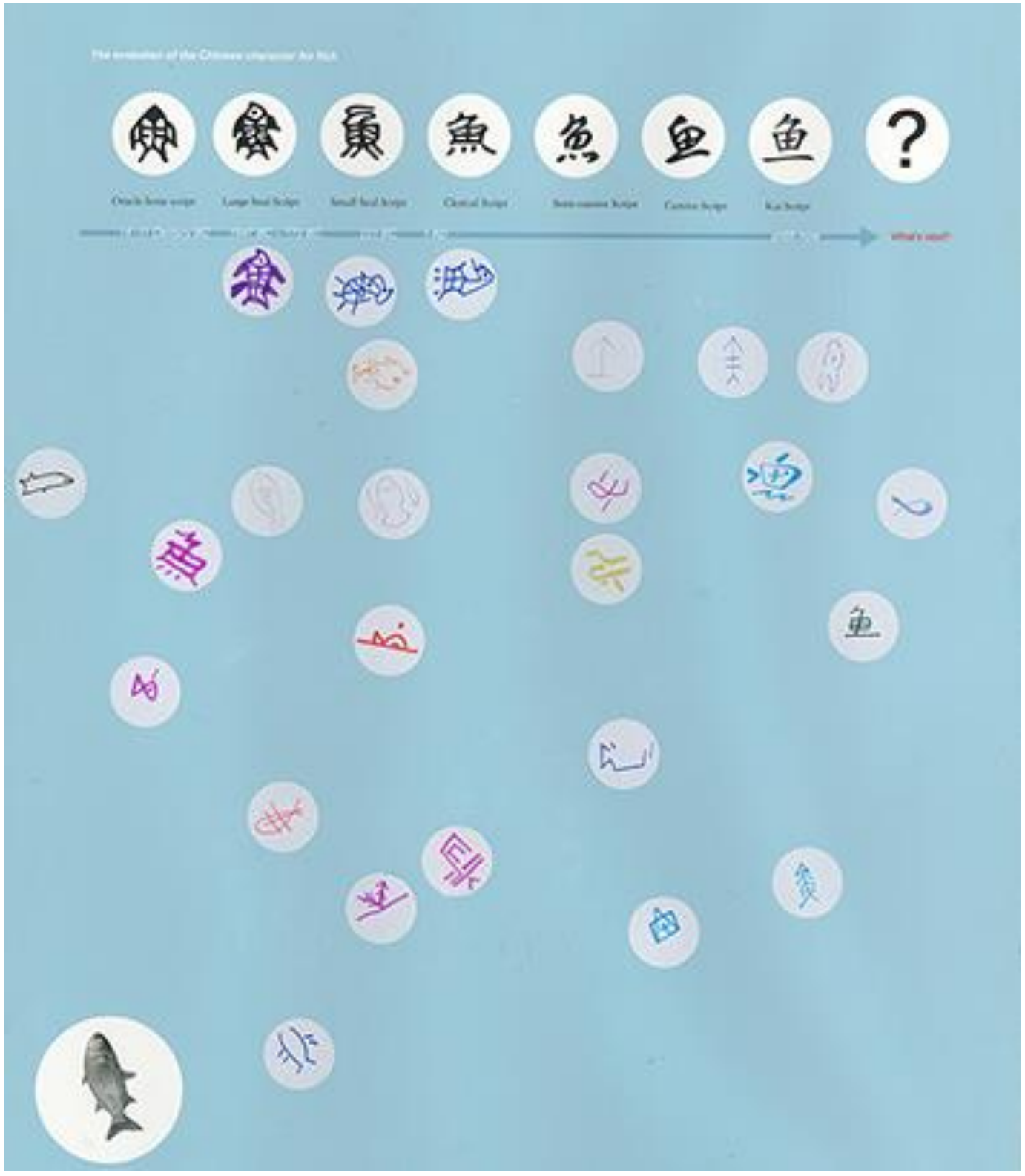




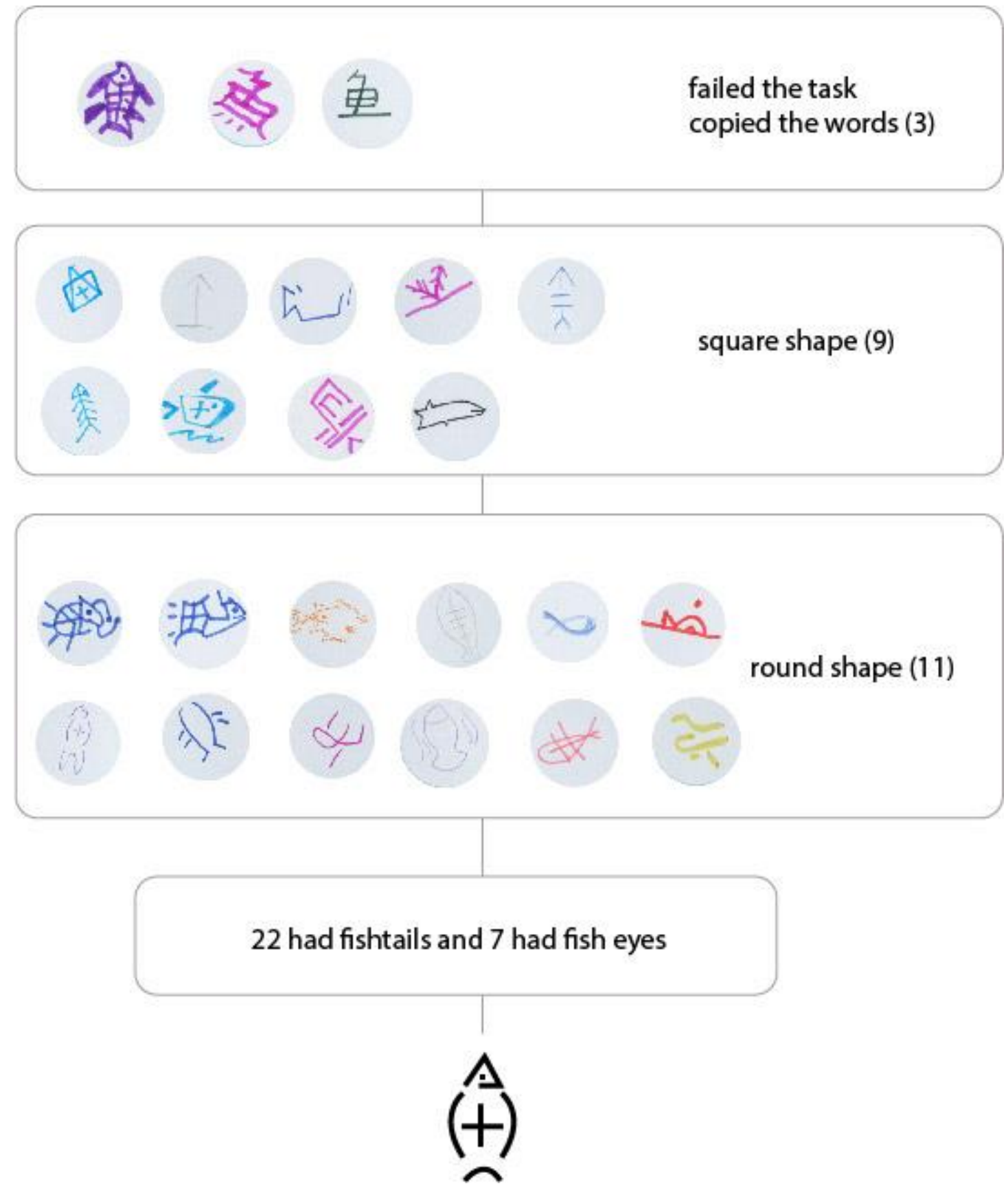

Figure 9: The fish poster with responses

Most Chinese characters of any period are square, however, among the total 25 fish in this exercise, 11 were rounder and 9 more square, 3were copied the words. Clearly, participants were influenced more by the shape of the animal than the shape of the character. 22 had fishtails and seven had fish eyes. Therefore, a more rhomboid body plus a fishtail are the main elements derived from these results and added into the final version of the fish design.

\section{Horse:}




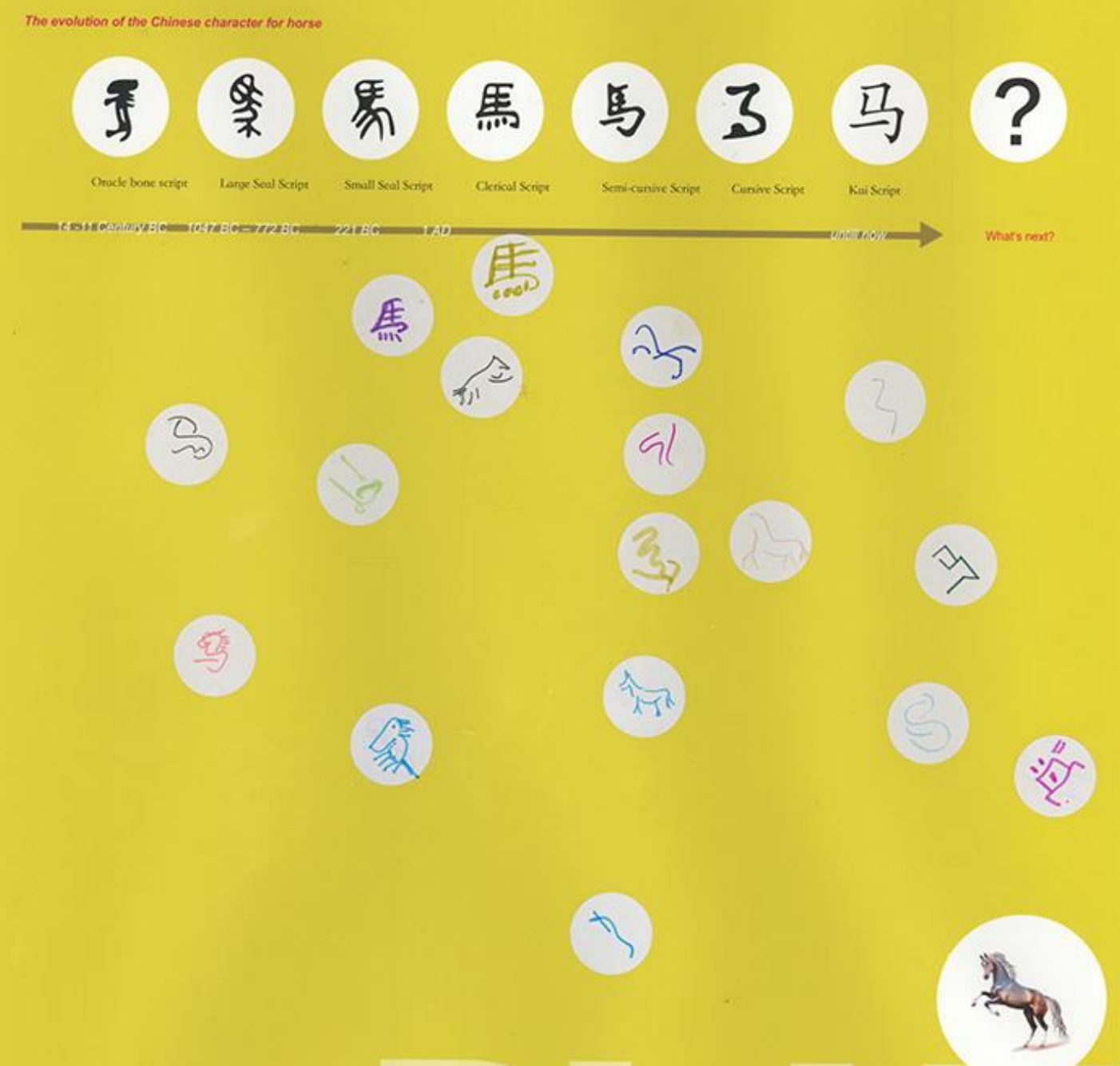




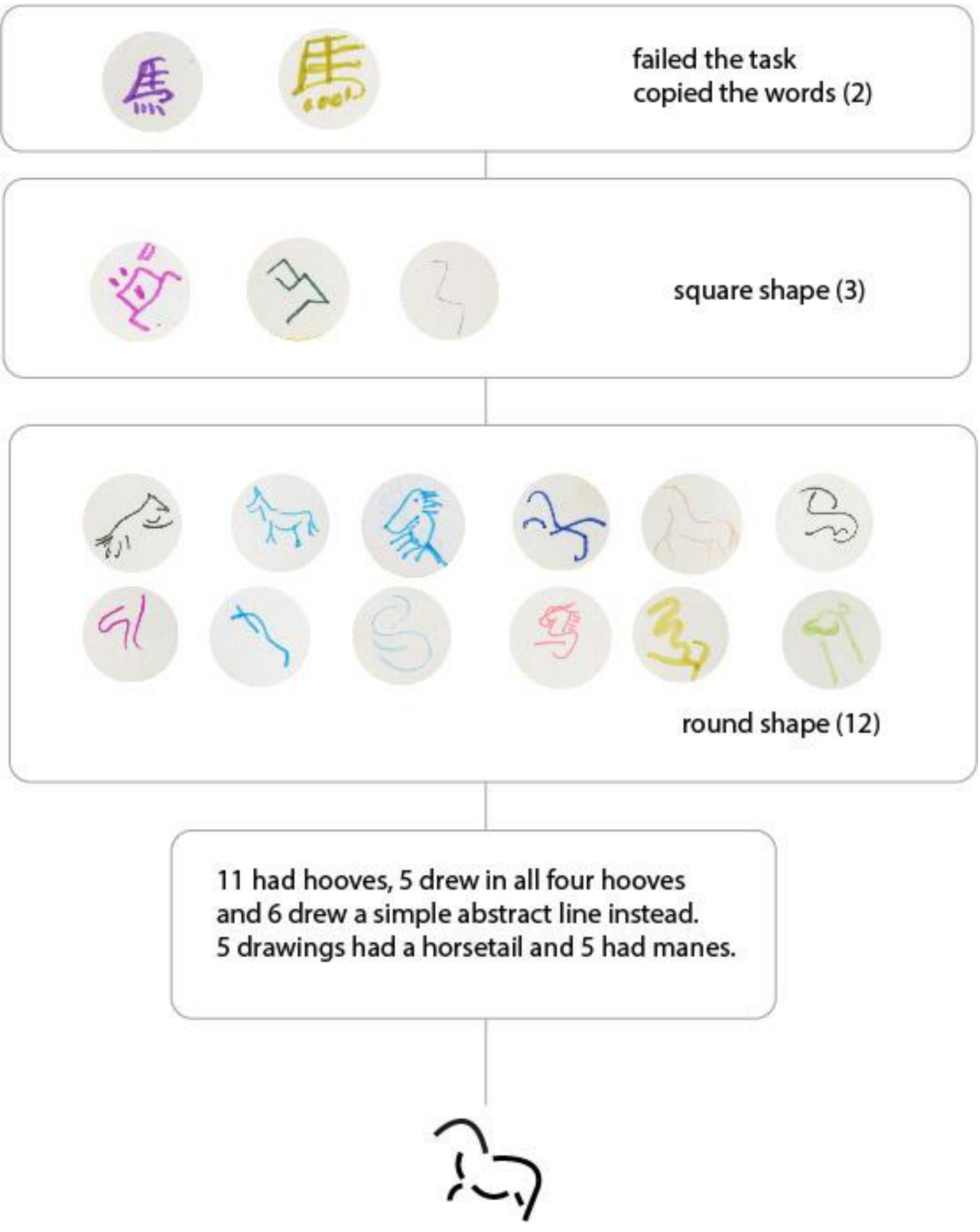

Figure 10: the horse poster with responses

Among all the 17 horses drawn, 5 were more square - similar to the character itself 12 were curved and therefore closer to the image. 11 had hooves, 5 drew in all four hooves and 6 drew a simple abstract line instead. 5 drawings had a horsetail and 5 had manes. A curved shape plus a hoof in some form are the main features - tails and manes were additional.

\section{Findings}


There were levels of interference with choice patterns caused by the expectations generated by cultural background. Shapes tended towards the least abstract and choices amongst Chinese participants seemed strongly culturally determined.

These four character drawings were inclined to be more rounded or curved. Interestingly - in terms of the evolution of Chinese characters - the tendency moved from rounded to square: modern Chinese characters are labelled "squared characters", and people are trained to write characters in a square, strokes are mainly divided between the vertical and horizontal axes. The qualities of a vertical/horizontal stroke are key criteria for judging Chinese calligraphy. In this exercise, people tended to draw rounded characters, closer to the shape of the animal. This finding made an essential contribution to the final design.

Local features and parts of the body could, therefore, easily represent the whole animal, and design needn't be either holistic or specific. When participants drew a new character, they may have unconsciously explored the relationships between strokes of the Chinese character and the shapes of the key animals.

A final discovery was in terms of the interference effects of cultural background on cognition and design. Among the total 106 character stickers completed by participants, 10 were written exactly as if they had been copied from the evolutionary process described earlier (see section 1). 6 participants did this because they misunderstood the task request, did not follow the draw and stick concept. 4 of these, however, were drawn by Chinese and Taiwanese who understood the instruction literally and thus failed creatively. Feedback from a Chinese participant signaled that, once he started to draw a new character - while he knew he was required to create something new - his hand would not obey his mind: he simply drew a familiar character despite that not being his intention. This automaticity may be because recognition of the Chinese character system is deeply embedded. Not only that, profound cultural familiarity could make any novel disturbance - a redesign or new character - too unusual to be casually practiced in this context. Design thinking and creativity may be at odds with automatic responses. It might be that sophisticated cultural baggage is not always positive, and sometimes could affect creativity or lateral thinking negatively. On the other hand, people who are not familiar with the cultural background or, alternatively, people who are not literate enough, may be better able to contribute to the design process. It is possible that a 
tabula rasa allows for fewer inhibiting interferences. Such individuals might be able to contribute more productively to outcomes guided by a responsive design methodology.

Based on these analyses and findings, there may be some insights to be gained in terms of cooperative research and user-centred design. These linked engagements, while limited in many ways, nevertheless allow this researcher to identify generative insights. To consider resemblances between characters and animal shapes; to find a balance between them and consider the theoretical bases necessary in the design of any effective modern typefaces.
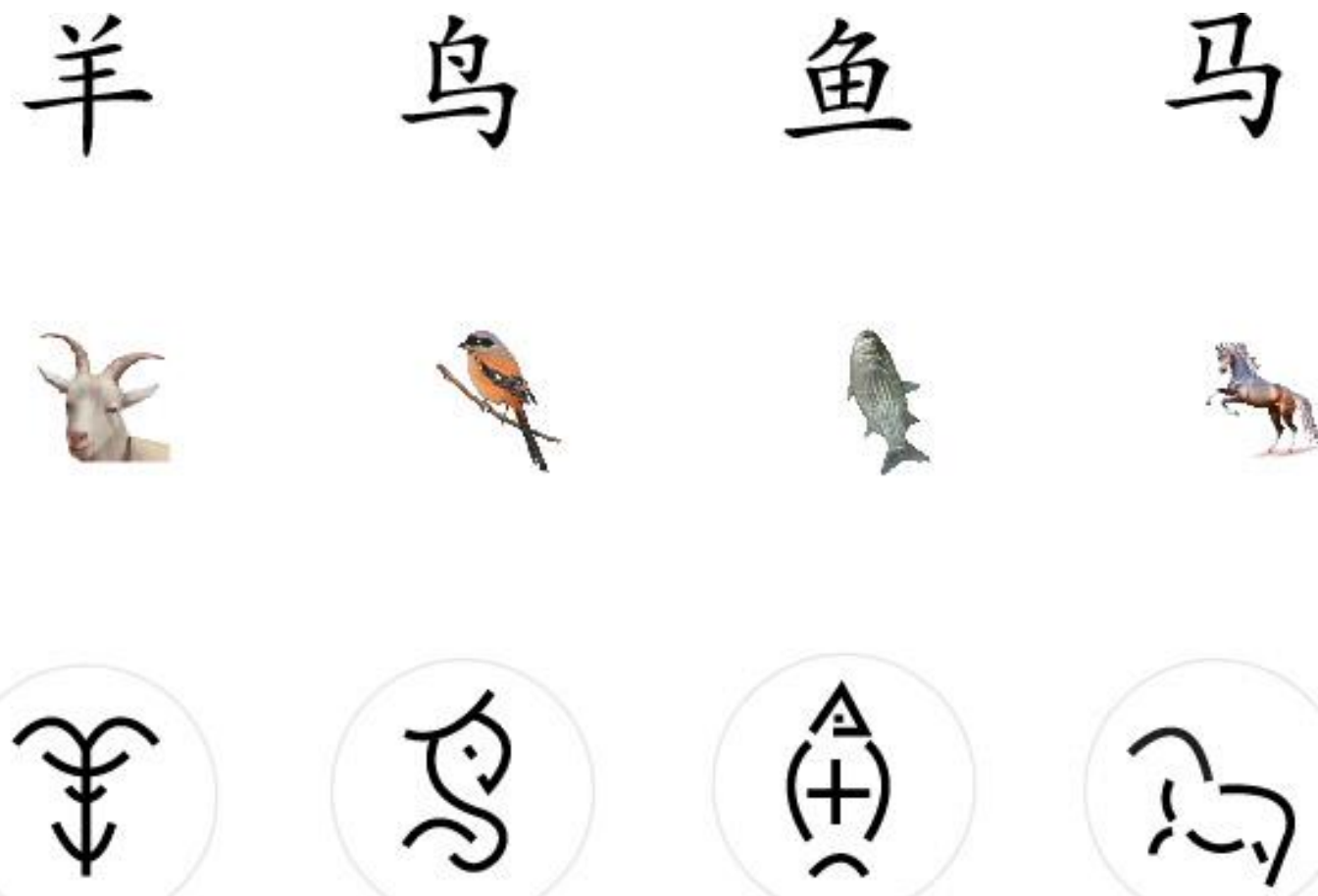

Figure 11: Final design

\section{Conclusion}

The RCA School of Communication Research Work in Process Show allowed an interesting short experiment that had some educative value for participants and provided a series of insights for the researcher. It is difficult to step outside one's culture, especially when that culture is as pervasive and rich as China's. An autoethnographer (Ruth et al (2018)) might well take the opportunity to situate themselves within the 
research process in an admission that subjectivity cannot be escaped and should therefore be embraced. A designer can occupy the next most productive space. By putting certain categories of user at the centres of both the research and associated design processes, and by a sensitive and responsive series of adaptive tests, much can be learnt about appropriate design theory, the cultural situation for any successful design and, finally, the possible shortcomings generated in part by interference of various kinds. What the researcher did was to prototype possible ways of thinking about how to engage with a wider public, within the overall discourse he is setting up. The researcher recognises that it was not a bona fide co-design process in terms of his research project: it was instead this was a way of getting some initial responses while addressing a question to see whether the kit - the tools designed - might be fit for purpose in terms of the doctoral methodology. This is a starting point - a catalyst - to see what the challenges might be. This is an enquiry currently being developed.

These experiments showed that, just as there is system interference between different Chinese character types (see section 1) there is also strong interference in terms of linguistic programming (see section 5). Design must recognise and indeed should make use of cultural and linguistic contingencies. The three experiments strongly point to user adaptability and also to the possibility that fewer language expectations - perhaps from non-native speakers, semi-literate or illiterate speakers - may allow for a positive and even creative design interface.

One limitation was the number of participants. Because participants were spontaneous and random (see section 3), the data collected was not precise enough to be theoretically rich. General conclusions can only be made due to the unknown cultural, racial and education backgrounds of participants. Secondly, it is unknown if participants are more or less influenced by the results of engagements with previous exhibitors.

This experiment has potential study value in terms of typefaces, icons, images and pictographs being different branches of visual communication. This was an attempt for typography to be better integrated with other visual elements and thus make typefaces more legible and acceptable. This could expand the typeface beyond its original function, contributing to other fields such as visual signs and instruction design which facilitate low literacy cognition.

Engaging in typeface design which is reader-oriented, participants become voluntary designers as part of the process. This method suggests that a visual designer 
should not only consider their users more, but only think about how to involve them more profoundly in design for an outcome that is open and adaptive.

\section{Bibliography}

Bachner, A. (2014). Beyond Sinology: Chinese Writing and the Scripts of Culture (New York, Columbia University Press)

Barnes, C.; Taffe, S. and Miceli, L. (2009). Multiple information failure: A case of different investments in form and content in graphic design. Visible Language, 43(2/3), pp.143-165.

Bennett, A. (2015). Connotative Localization of an HIV Prevention Image to Promote Safer Sex Practices in Ghana. Visible Language, 49(1/2), pp.25-38.

Bo, S. (2003). Between Heaven and Earth: A History of Chinese Writing (translated by Sherab Chödzin Kohn) (London, Shambhala)

Bunke, H. (2000). Handbook of character recognition and document image analysis. Singapore: World Scientific.

Guo, W. (2005). Image Design of Chinese Character. Zhuangshi, 146(6), pp.

Han, J. (2012) Chinese Characters (Cambridge, Cambridge University Press)

Lewi, P.J. (2006) Speaking of Graphics: An Essay on Graphicity in Science, Technology and Business at http://www.datascope.be/sog.htm (accessed 04/01/2018)

Li, CL. (2010). Unity and Variety: A Study of the Chinese Language and its Cultural Implications. Unpublished dissertation, UC San Diego at http://www.escholarship.org/uc/item/8tt1c8sd (accessed 02/01/2018)

Li, CX. (2003). On the Introspection of Simplifying Chinese Character and Its New Thinking. Journal of Jiangxi Normal University, 36(4), pp.72-76

Li, XJ. (2015). Pre-Qin Ancient Chinese Characters and the Origins of Chinese Calligraphy. 先秦古文字与中国书法的源头李祥俊 in 衡水学院学报, Issue 1, pp.121-125

Neurath, O. (2010). From Hieroglyphics to Isotypes: A Visual Autobiography (London, Hyphen Press)

Noël, G. (2015). Designing a Visual Tool to Interview People with Communication Disabilities: a user-centered approach. Visible Language, 49(1/2), pp.62-79.

Norman, J. (1988). Chinese (Cambridge, Cambridge University Press)

Park, H. (2016) The Writing System of Scribe Zhou: Evidence from Late PreImperial Chinese Manuscripts and Inscriptions (5th-3rd Centuries BCE) (Berlin, De Gruyter)

Paulovich, B. (2015). Design to improve the Health Education Experience: using participatory design methods in hospitals with clinicians and patients. Visible Language, 49(1-2), pp.144-159 
Qian, C. (2013). Analysis and Suggestions to Pros and Cons of Chinese Characters Simplification. Journal of West Anhui University,29(3), pp.131-132

Ruth, D.; Suze Wilson; Ozan Alakavuklar and Andrew Dickson (2018). Anxious academics: talking back to the audit culture through collegial, critical and creative autoethnography. Culture and Organization, 24(2), pp $154-170$.

Song, H. and Schwarz, N. (2009). If It's Difficult to Pronounce, It Must Be Risky:

Fluency, Familiarity, and Risk Perception. Psychological Science, 20(2), pp 135-138

Stauss, A and Zender, M (2017). Design by Consensus: A New Method for Designing Effective Pictograms. Visible Language, 51(2), pp.7-33

Wang, H.F. (2010). The Appropriateness of Icon Representations for Taiwanese Computer Users. Visible Language, 44(3), pp. 305-329

Wang, N. (2010). A Historical Account of the Controversies over the Use of Simplified or Unsimplified Chinese Characters and Their Standardization. Journal of Yunnan Normal University, 42(6), pp. 1-6

Zender,M.(2017). DrawIt: a user-drawn design research method for symbol design. Visible Language, 51(2), pp.34-61.

Zender, M. and Cassedy, A. (2014). (mis)understanding: icon comprehension in different cultural contexts. Visible Language, 48(1), pp.69-95.

\section{About the author}

Wenbo Ai currently is an Mphil student at RCA, he studied his BA in Visual Communication Design at Hubei Institute of Fine Arts in China, and MA in Service design Innovation at University of Arts, London/ London College of Communication. He was a graphic designer at Design Against Crime Research Centre Central Saint Martins (University of Arts, London).

wenbo.ai@network.rca.ac.uk 